\title{
Umbilical hernia in autosomal dominant polycystic kidney disease
}

\author{
Yoshinari Hattori $\cdot$ Junichi Hoshino $\cdot$ Tatsuya Suwabe $\cdot$ \\ Keiichi Sumida $\cdot$ Koki Mise $\cdot$ Noriko Hayami · \\ Naoki Sawa $\cdot$ Kenmei Takaichi $\cdot$ Yoshifumi Ubara
}

Received: 25 November 2013/Accepted: 11 December 2013/Published online: 10 January 2014

(C) The Author(s) 2014. This article is published with open access at Springerlink.com

Keywords Umbilical hernia $\cdot$ Autosomal dominant polycystic kidney disease

A 44-year-old woman was diagnosed with autosomal dominant polycystic kidney disease. Her mother has the same disease. Even after hemodialysis was started in 2003 due to end-stage renal failure, abdominal distention progressed and a protruding umbilical hernia became prominent (Fig. 1a, b). However, the surgeons hesitated to perform hernia repair. Transcatheter arterial embolization (TAE) was performed to treat massive hepatomegaly in 2005 [1] and to treat bilateral nephromegaly in 2006 [2]. Her abdominal distension and umbilical hernia both improved in 2013 (Fig. 2a, b). This case emphasizes that massive polycystic liver and kidneys may contribute to umbilical hernia formation by increasing the intraabdominal pressure.

Acknowledgments This study was funded by the Okinaka Memorial Institute for Medical Research.

Conflict of interest All authors report no conflicts of interest.

Open Access This article is distributed under the terms of the Creative Commons Attribution License which permits any use, distribution, and reproduction in any medium, provided the original author(s) and the source are credited.

Y. Hattori - J. Hoshino $\cdot$ T. Suwabe $\cdot$ K. Sumida $\cdot$ K. Mise $\cdot$

N. Hayami $\cdot$ N. Sawa $\cdot$ K. Takaichi $\cdot$ Y. Ubara $(\bowtie)$

Nephrology Center, Toranomon Hospital, 2-2-2 Toranomon,

Minato, Tokyo 105-8470, Japan

e-mail: ubara@toranomon.gr.jp

Y. Ubara

Okinaka Memorial Institute for Medical Research, Toranomon

Hospital, Tokyo, Japan 
Fig. 1 a Gross appearance of pre-TAE. b Gross appearance of post-TAE. Arrow shows protruded umbilical hernia
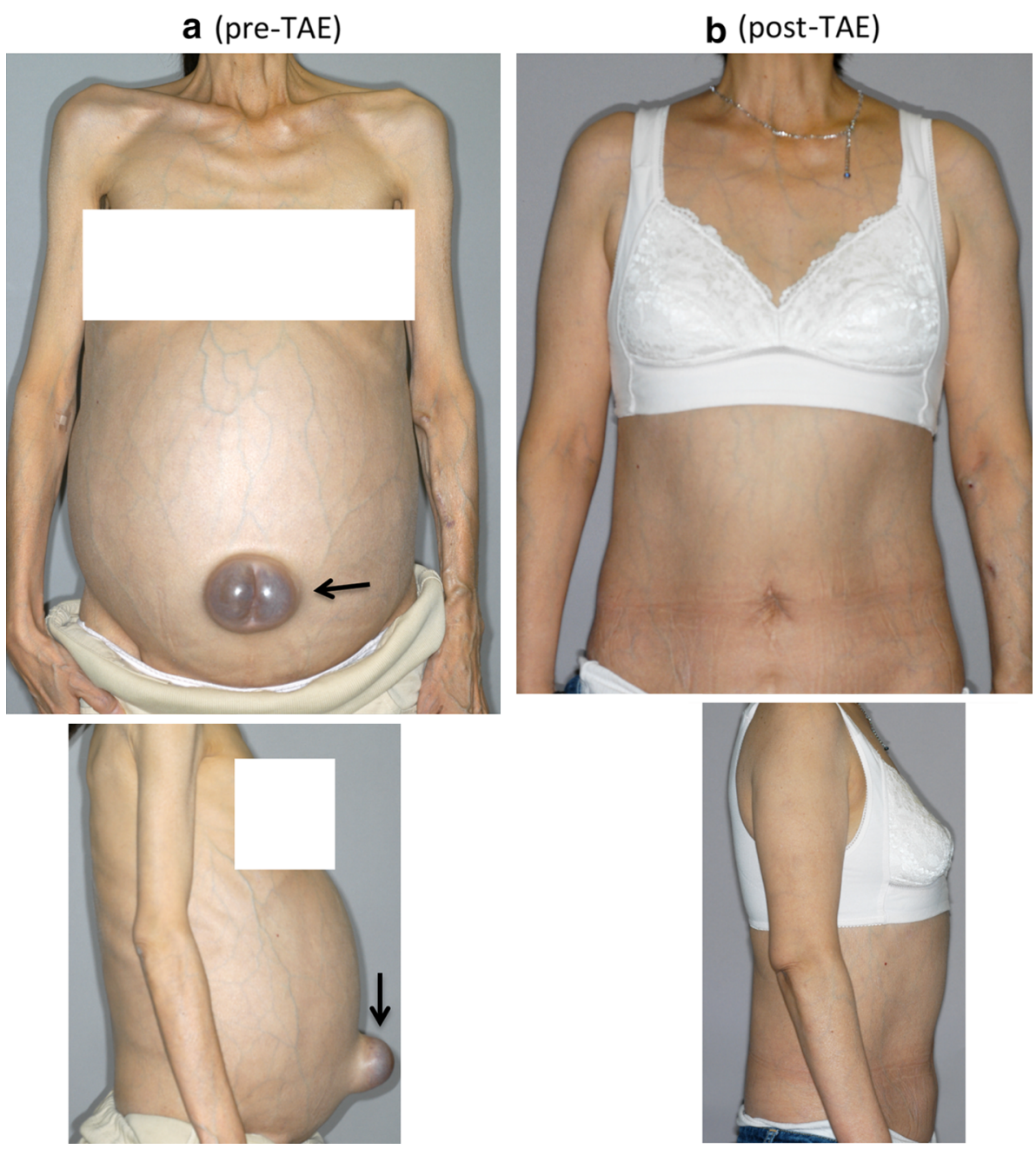
Fig. 2 a Computed

tomography images pre-TAE.

b Computed tomography

images post-TAE. Arrow shows

protruded umbilical hernia
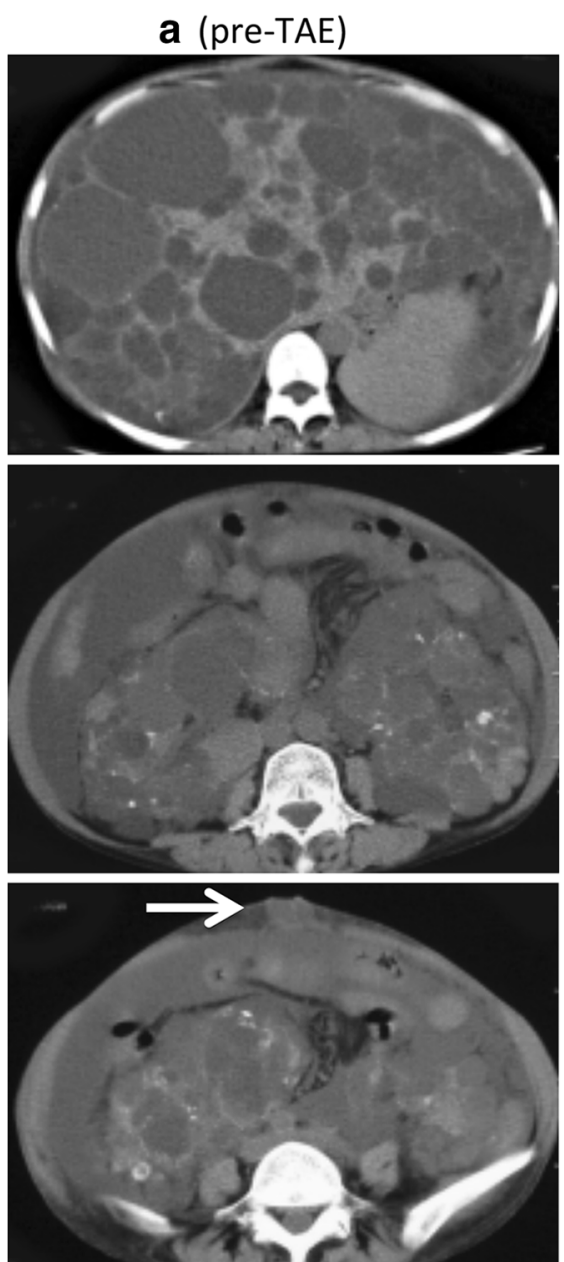

b (post-TAE)
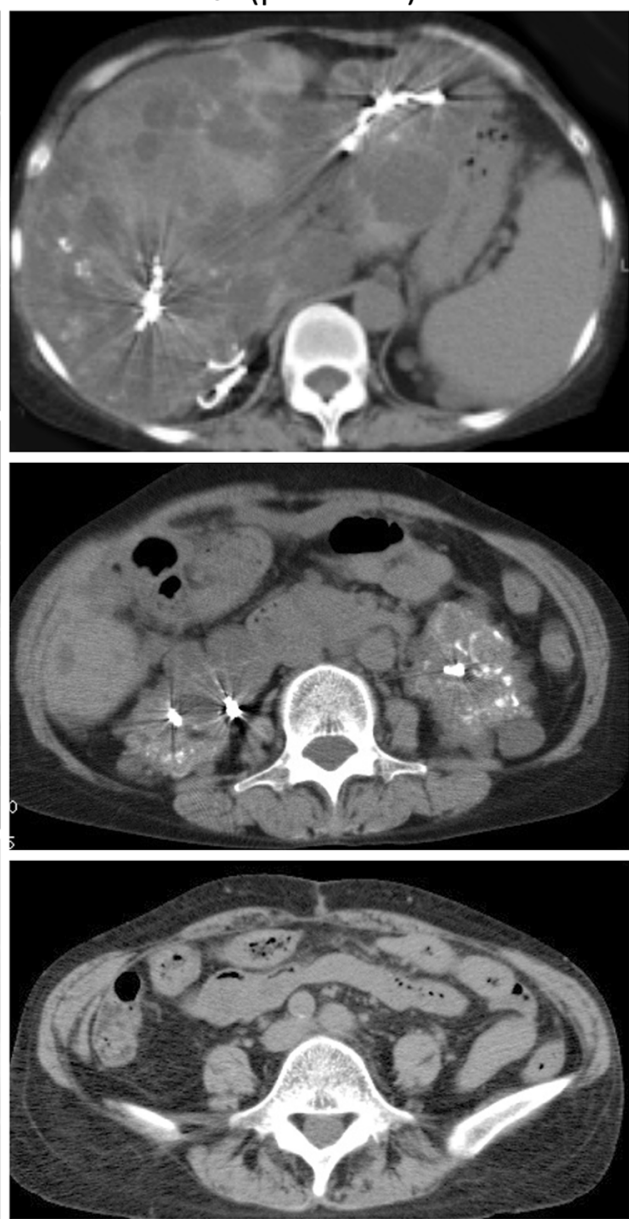

\section{References}

1. Takei R, Ubara Y, Hoshino J, Higa Y, Suwabe T, Sogawa Y, Nomura K, Nakanishi S, Sawa N, Katori H, Takemoto F, Hara S, Takaichi K. Percutaneous transcatheter hepatic artery embolization for liver cysts in autosomal dominant polycystic kidney disease. Am J Kidney Dis. 2007;49(6):744-52.
2. Ubara Y, Tagami T, Sawa N, Katori H, Yokota M, Takemoto F, Inoue S, Kuzuhara K, Hara S, Yamada A. Renal contraction therapy for enlarged polycystic kidneys by transcatheter arterial embolization in hemodialysis patients. Am J Kidney Dis. 2002;39(3):571-9. 